\title{
Pathology as the enabler of human research
}

\author{
James M Crawford ${ }^{1}$ and Mark L Tykocinski ${ }^{2}$ \\ ${ }^{1}$ Department of Pathology, Immunology and Laboratory Medicine, University of Florida College of Medicine, \\ Gainesville, FL, USA and ${ }^{2}$ Department of Pathology and Laboratory Medicine, University of Pennsylvania, \\ Philadelphia, PA, USA
}

\begin{abstract}
Academic Pathology is a key player in human molecular science and in the powerful initiatives of the National Institutes of Health. Pathologists generate data crucial to virtually every molecular study of human tissue, and have the necessary skills and authority to oversee processing of human tissues for research analysis. We advocate that Academic Pathology is optimally positioned to drive the molecular revolution in study of human disease, through human tissue collection, analysis, and databasing. This can be achieved through playing a major role in human tissue procurement and management; establishing high-quality 'Pathology Resource Laboratories'; providing the scientific expertise for pathology data sharing; and recruiting and training physician scientists. Pathology should position itself to be the local institutional driver of technology implementation and development, by operating the resource laboratories, providing the expertise for technical and conceptual design of research projects, maintaining the databases that link molecular and morphological information on human tissues with the requisite clinical databases, providing education and mentorship of technology users, and nurturing new research through the development of preliminary data. We also consider that outstanding pathology journals are available for the publication of research emanating from such studies, to the benefit of the pathology profession as an academic enterprise. It is our earnest hope that Academic Pathology can play a leading role in the remarkable advances to be made as the 21st century unfolds. Laboratory Investigation (2005) 85, 1058-1064. doi:10.1038/labinvest.3700321; published online 1 August 2005
\end{abstract}

Keywords: pathology; human tissue; tissue banking; genomics; proteomics; molecular imaging

\section{The trumpet call}

For most of human history, the earth was regarded as the center of the universe. In AD1543, Nicolaus Copernicus published De revolutionibus orbium coelestium, declaring that the earth is not the center of the cosmos, but revolves around the sun. Biomedical science now faces its own Copernican revolution. From the days of Aristotle through the second-half of the 20th century, the investigatordriven hypothesis resided at the center of the scientific universe. This paradigm entailed a Principal Investigator generating a hypothesis, collecting data to test the hypothesis, and publishing results and interpretation in the framework of that hypothesis. Arguably, the investigator collected no more (and no less) data than required to test the hypothesis. This may no longer be true.

Correspondence: Dr JM Crawford, MD, PhD, Department of Pathology, Immunology and Laboratory Medicine, University of Florida College of Medicine, PO Box 100275, Gainesville, FL 32610-0275, USA.

E-mail: crawford@pathology.ufl.edu

Received 17 June 2005; accepted 22 June 2005; published online 1 August 2005
We have entered into an era in which our ability to generate data far outstrips our ability to generate hypotheses, let alone test them with immediacy. Given the growing array of high-throughput technologies available for obtaining vast data libraries for the genome, transcriptome, proteome, metabolome, lipidome (and so on), data is now beginning to supplant the hypothesis at the center of the scientific universe. Data can be collected-and published-as a stand-alone product. The corollary is that investigators can now mine existing data libraries in silico, without necessarily having to generate their own primary data. The paradigm shift required for review of submitted research grants on the one hand, and of manuscripts submitted for publication on the other, presents a challenge to all individuals involved in the scientific review process.

The National Institutes of Health is embracing the stunning increase in scientific data generation through the well-publicized and extensively discussed NIH Roadmap (vis: www.nihroadmap.nih. gov). The programmatic initiatives under the Roadmap 'New Pathways to Discovery' speak well to this point: Technology for Molecular Analysis; Molecular Libraries and Molecular Imaging; Structural 
Biology; Bioinformatics and Computational Biology; Nanotechnology and Nanomedicine. Each of these programmatic initiatives requires departure from the parochial environment of localized Principal Investigator-driven laboratories, so as to encompass much larger scientific research teams operating across multiple disciplines. Hence, the Roadmap declares that 'Research Teams of the Future' must include 'Comprehensive Multidisciplinary Teams' performing 'High-Risk Research'.

Accompanying this emerging prominence of team-driven data acquisition is a new-found appreciation for the importance of human tissue. While experimental research with animal models of human disease remains a key foundation for the biomedical scientific enterprise, special emphasis is being given to molecular analysis of human tissues. Acceleration of translational research, that is, human tissue-based, is now seen as pivotal for 'reengineering the clinical research enterprise'. The question can thus be asked: what is the role of Academic Pathology in this new scientific era?

We suggest an answer. Academic Pathology must strive to drive the agenda for human tissue collection, analysis, and databasing. This trumpet call is not new. At the 2000 Summer Meeting of the Association of Pathology Chairs (Boulder, CO, USA), Leo $\mathrm{T}$ Furcht, MD made the following statements. First, pathologists generate data crucial to virtually every molecular study of human tissue. Academic pathologists have the necessary skills and authority to oversee processing of human tissues for research analysis. The authority, in particular, cannot be delegated. Second, Academic Pathology is optimally positioned to drive the molecular revolution in study of human disease. Academic Pathology is the starting point for virtually everything the translational research enterprise seeks: expertise in handling of human tissues; knowledge about human disease; and the technological innovations required to advance the scientific study of human disease. However, Academic Pathology can only keep the mountaintop by being aggressively proactive. Dr Furcht closed by stating, 'If you are not part of the steam-roller, you are part of the road.'

So what has changed in the ensuing 5 years? The publication of the Human Genome, vast increases in data connectivity and processing, and ever-increasing international cooperation in the biomedical research enterprise are only part of the changes. The NIH annual budget was doubled, thereby increasing expectations for translational outcomes. However, Congressional and public support of the biomedical research enterprise has soured a bit. ${ }^{1}$ Concern has been raised about the scientific culture of arrogance wherein continued major increases in funding are expected, about conflicts-of-interest in academic:corporate relationships, and about the need to direct public resources elsewhere such as the 'war on terrorism'. Biomedical science has received the message that this is the 'end of the pig trough'. Against this somewhat discouraging backdrop is our acute awareness that we now have unprecedented opportunities for breathtaking scientific progress. The trumpet call remains. Can the recent public investment in biomedical research pay off? Will translational outcomes be forthcoming?

Academic Pathology has a unique opportunity to advance the public interest. Indeed, Greg Downing, $\mathrm{PhD}$, of the National Cancer Institute Office of the Director, has stated that, 'The NIH desperately needs input from the Pathology Community' (personal communication, June, 2004). Specifically, human tissue and the data therefrom have become 'the currency of the realm'. The argument can be made that Academic Pathology should oversee not only high-quality tissue procurement and processing, but also: high-throughput molecular analysis; comprehensive databasing; and pan-national management of information. Pathologists by their very profession are data managers for human disease. Application of their considerable skills to the research enterprise is an imperative.

Carolyn Compton, MD, the new Director for the Laboratory for Human Specimen Banking Research at the National Cancer Institute, states that, 'Billions of dollars will be spent analyzing the 'Cancer Genome'. That means that human tissue samples are worth their weight in platinum' (Association of Pathology Chairs meeting, July, 2004, Mont Tremblant, Quebec, Canada). We have examined the prices of precious metals on the US Mercantile Exchange, and consider that Dr Compton underestimates the value of human tissue samples. The price of platinum at the time of the writing of this editorial (May 17, 2005) was $\$ 22.86 / g$. The price of gold was $\$ 11.00 / \mathrm{g}$, and of crude uranium $\$ 0.06 / \mathrm{g}$. We observe that patient hospitalization for resection of a few grams of tumorous tissue can easily cost over \$10 000. We estimate that the cost of harvesting and storing aliquots of the tumorous tissue for research purposes approaches $\$ 1000 / \mathrm{g} /$ year. The cost of database management for alignment of clinical information with the tissue information, and the distribution costs for tissue delivery, can add another $\$ 1000 / \mathrm{g} /$ year. We therefore conclude that the value of human tumor tissue is at least 100 -fold the price of platinum. The price of diamonds— $\$ 5000 / g —$ seems about right.

\section{Human tissue procurement}

Our proposed strategy for Academic Pathology serving the public interest is for it to (1) play a major role in human tissue procurement and management; (2) establish high-quality 'Pathology Resource Laboratories'; (3) provide the scientific expertise for pathology data sharing; and (4) recruit and train physician scientists.

There are an estimated 300 million human tissue specimens stored in repositories throughout the 
United States. ${ }^{2}$ An estimated 20 million additional specimens are added each year, without or with annotated clinical information. The National Cancer Institute currently provides approximately $\$ 40$ million annually to fund human tissue repositories, largely through such mechanisms as SPORE grants (as for prostate, breast, and lung cancers); direct human tissue banking; and NCI-funded cancer centers. Nonprofit organizations such as the $\mathrm{Al}$ pha-1 Foundation and Alzheimer's Foundation also fund human tissue repositories. Corporate repositories also exist, often in a for-profit mode.

The National Cancer Institute has participated in the development of a new concept for a pan-national system of human tissue procurement, in the posting of 'Biorepositories for 21st Century Medicine: Pathway to a National Biospecimen Network'. ${ }^{3}$ These objectives are: to create a molecular taxonomy for cancer; to create new targeted therapies; to identify new uses for existing targeted drugs; and to accelerate the era of personalized medicine. Table 1 gives the specific strategies for achieving these goals. The NCI collaborated with a not-for-profit organization, C-Change (formerly the National Dialogue on Cancer) to develop the National Biospecimen Network Blueprint, which outlines a vision for a biorepository network specifically designed to support highthroughput research. ${ }^{2}$ It is hoped that a harmonized national tissue procurement effort will promote standardized collection of biospecimens, and hence facilitate the effort to refine diagnostics so as to identify high- and low-risk disease; find novel molecular targets for therapies; find clinically useful biomarkers by identifying correlations between gene expression profiles and treatment responders; and select the appropriate patients for targeted therapies. This effort dovetails with the 'caBIG: Cancer Biomedical Informatics Grid' (https://cabig.nci.nih. gov/in_the_news;). ${ }^{4}$ The 'caBIG' initiative declares that cyberinfrastructure is a 'third way' in Biomedical Research, with the goal of linking databases and investigators throughout the biomedical research world. This is intended to promote molecular-based medicine based on tissue analysis, linking extensive database repositories with superb file-transfer-

Table 1 Vision for tomorrow: a national network of biorepositories

\begin{abstract}
Large numbers of high-quality, clinically annotated samples Diversity of cancer types and populations

Pathology and clinical (including longitudinal) annotation of specimens

Access through a centralized peer-review process

Management of ethical and legal issues for a chain of trust Resources provided without intellectual property restrictions Best practices-based SOPs for reproducible results Bioinformatics infrastructure for storing research results and building in silico capability
\end{abstract}

SOPs: standard operating procedures.

Source: Julie Schneider, DPhil, Technology Program Manager, National Cancer Institute. protocol (FTP) capabilities. Using an open-source, open-access data network, the hope is to break down barriers between fiercely independent organizations and institutions, and move biomedical research through the multidisciplinary crossroads into the new era of data generation and utilization.

The appointment of Dr Carolyn Compton, one of the world's leading pathologists, to direct the Laboratory for Human Specimen Banking Research within the National Cancer Institute is highly opportune. We encourage the extramural community of Academic Pathology to use every opportunity to work with the National Institutes of Health to advance human tissue procurement.

\section{The pathology resource laboratory}

After human tissue procurement, molecular and morphological analysis of these tissues constitutes the next critical step in advancing the scientific agenda. We submit that pathology-based centralized core laboratories are much preferable to distributed, niche subspecialty diagnostic laboratories. Through centralized laboratories, high-throughput analysis can be provided for genomics and proteomics (for starters), and for molecular imaging. These laboratories can then provide for data management-both of the tissue analytes and the clinical datasets. It is then reasonable to posit that the interventional strategies of cellular, gene, and protein therapeutics may be linked, and possibly driven, by these self-same pathology resource laboratories. Beyond the question of who will collect and bank the human tissue, the key questions are: who will generate the highthroughput molecular data; and who will interpret it?

We submit that Academic Pathology should position itself to be the local institutional driver of technology implementation and development, and thereby to be the manager of the crossroads of bedside-to-bench-to-bedside research. The application of expertise is in:

- Performing high-throughput molecular analysis: genomics; transcriptomics; proteomics.

- Coupling high-resolution imaging to advanced molecular diagnostics, thereby leading towards 'molecular maps' pegged to tissue architecture.

- Handling of large analytical and clinical datasets.

- Long-term storing and accessing of data.

- Managing the regulatory environment for human research.

- Offering the added value of the pathologist/ scientist as collaborator.

The last point bears emphasis: human tissue procurement and analysis is not a commodity provided by the pathologist. The pathologist is a scientific collaborator as well. Specifically, the pathologist as 'resource scientist' can:

- Address both technical and conceptual design of research projects. 
- Provide advanced instrumentation and consultative support.

- Consult right from the initial conceptualization of projects, and educate technology users.

- Support hypothesis-testing and data-generating experiments.

- Act as mentor, through the training of client scientists and their own trainees.

- Nurture new research, by being proactive in development of new preliminary data.

The fundamental premise is that the Pathology Resource Laboratory can provide investigators access to high-quality, cost-effective advanced technologies; and provide the scientific expertise necessary to use these technologies effectively. With the likelihood that successful research programs will increasingly demand parallel development of resource and research laboratories, pathologists will thus find themselves at the crossroads of the academic research enterprise.

Pathology departments are ideally positioned to serve as institutional drivers of shared resource laboratories that pioneer and implement high-end technologies. It is a natural step for such departments to leverage their clinical laboratory oversight expertise in promoting research-oriented resource laboratories. In this way, Pathology departments can emerge as institutional hubs for the gamut of centralized laboratory services within an academic center, spanning both clinical diagnostics and research support. Resource laboratory services can encompass multiplexed molecular and cellular diagnostics, biomedical imaging, cell processing, tissue banking, high-throughput functional screening, biomedical informatics, and beyond.

\section{Pathology research: the finished product}

NIH-funded extramural awards to academic medicine in the United States are public information (http://grants2.nih.gov/grants/award/trends/medschc. $\mathrm{htm})$. Award data for fiscal year 2004 were available for review. Table 2 shows the leading clinical and basic science departments garnering extramural funding from the NIH. Departments of Internal Medicine garner by far the greatest number of awards and award dollars. Departments of Pathology, Pediatrics, Microbiology/Immunology, Biochemistry and Pharmacology are closely clustered, representing the next major group of funded departments. In all, medical schools receive approximately half of the NIH budget. Departments of Pathology receive $1 / 45$ of the NIH budget $(2.54 \%)$, or about $1 / 20$ of all NIH-funded extramural research. These data do not include awards to Departments of Pathology in independent hospitals, and so the final numbers may be slightly higher. One may conclude that Academic Pathology is well represented as a discipline in academic medicine. Whether a loftier status should be attained as a result of new NIH initiatives is a matter of speculation.

Consideration can now be given to the product of pathology research. A fundamental goal of pathology research is to understand the structural and molecular basis of human disease. In particular, effort is made to advance understanding of human disease so as to: improve our diagnostic abilities; enable the best possible clinical management through clinical decision making and accurately targeted treatment; enable effective monitoring of disease course, treatment effectiveness, and complications; and identify novel opportunities for treatment of human disease. At the risk of oversimplification, the operational goals of investigative pathology are given in Table 3 . There is a clear role for crosstalk between experimental studies with animals and analysis of human tissues. There is also a clear role for navigation between in vivo and in vitro research, to which we should now also add in silico research. Examples of how these goals translate into published articles are given in Table 4 .

Although the examples in Table 4 are listed (topto-bottom) as a function of decreasing impact, we consider that there is a place for all such forms of publication. From the bottom, descriptive case

Table 2 Extramural NIH Funding, 2004 ${ }^{\mathrm{a}}$

\begin{tabular}{lrrr}
\hline & \# Grants & Total award & \% of NIH budget \\
\hline Departments of Internal Medicine & & & \\
Departments of Pathology & 6627 & $\$ 3,082,600,819$ & \\
Departments of Pediatrics & 1634 & $\$ 620,257,860$ & 2.21 \\
Departments of Surgery & 1476 & $\$ 605,256,042$ & 2.16 \\
Departments of Microbiology/Immunology & 1763 & $\$ 65,137,666$ & 1.12 \\
Departments of Biochemistry & 1760 & $\$ 595,083,550$ & 2.33 \\
Departments of Pharmacology & 1486 & $\$ 491,679,171$ & 2.12 \\
Departments of Anatomy and Cell Biology & 1297 & $\$ 463,495,672$ & 1.75 \\
Departments of Genetics & 799 & $\$ 407,805,771$ & 1.65 \\
All Medical Schools & 27886 & $\$ 11,247,579,569$ & 1.46 \\
All Independent Hospitals & 3784 & $\$ 1,567,654,215$ & 40.13 \\
\hline
\end{tabular}

${ }^{\mathrm{a} S}$ Selected academic departments only.

Source: http://grants2.nih.gov/grants/award/trends/medschc.htm. 
Table 3 General goals of pathology research

Human research

Gene/protein identification, characterization, determination of causality

Global gene expression, tissue identification, correlations with outcomes

In vitro cellular models, molecular/physiologic/structural exploration

Technical advances: diagnostics, analysis, applications

Advancement of ability of pathologists to understand and diagnose disease

Advancement of ability of health care providers to treat disease

Animal research

Establishment and characterization of animal models of human disease

Gene/protein identification, characterization, determination of causality

In vitro cellular models, molecular/physiologic/structural exploration

Exploration of molecular and structural pathogenesis of disease

Table 4 Examples of pathology research on human tissue

Genome-wide hypothesis-testing and validation

In vivo $\leftrightarrow$ in vitro mechanistic investigation

Molecular exploration and hypothesis generation

Rigorous technical analytical advances

Molecular correlations and clinical outcomes

Case series with molecular correlations

Correlative case series

Descriptive case studies

studies provide critical documentation of events that can occur in humans, however rare or unexplained. This is the first step in exploring the pathogenesis of human disease. Correlative case series provide more robust documentation of disease processes in humans, and often are of high value for the practice of diagnostic pathology. Hypotheses and potential answers are generated through the next categories of reports, namely case series with molecular data and correlations, and molecular data correlated with clinical outcome. We consider that robust and rigorous technical advances for molecular analysis of tissues have their own broad impact. It is only with such advances that the top group of mechanistically oriented studies can be performed: molecular exploration (of extant databases or of new data) with hypothesis generation; definitive mechanistic studies that take advantage of both in vivo and in vitro experimental systems, and ultimately genome-wide hypothesis testing and validation. While these investigative groupings are not meant to be exclusive, perusal of the published pathology literature can identify excellent examples of each. As Laboratory Investigation is a mechanistically oriented pathology journal, several outstanding examples of the upper-tier articles come to mind..$^{5-9}$

Where pathology research is published then becomes of interest. Table 5 gives the Impact Factors and Immediacy Index for three general groups of
Table 5 Examples of Journal Impact Factors ${ }^{\mathrm{a}}$ and Immediacy Index

\begin{tabular}{lcc}
\hline Journal & $\begin{array}{c}\text { Impact factor } \\
\text { 2004 }\end{array}$ & $\begin{array}{c}\text { Immediacy index } \\
\text { 'High impact' journals }\end{array}$ \\
Ann Rev Immunol & & \\
New Eng J Med & 52.431 & \\
Nature & 38.570 & 10.478 \\
Science & 32.182 & 6.089 \\
Cell & 31.853 & 7.379 \\
J Am Med Assoc & 28.389 & 7.632 \\
J Exp Med & 24.831 & 5.499 \\
J Clin Invest & 14.588 & 2.436 \\
& 14.204 & 2.554 \\
'Tissue/organ-specific' journals & & \\
Neuron & 14.439 & \\
J Natl Cancer Inst & 13.856 & 2.672 \\
Gastroenterology & 13.092 & 3.031 \\
Circulation & 12.563 & 2.529 \\
Hepatology & 10.416 & 1.758 \\
Diabetes & 8.848 & 1.599 \\
Brain & 8.201 & 1.443 \\
J Am Soc Nephrol & 6.644 & 1.221 \\
& & 1.080 \\
'Pathology' journals & & \\
Am J Pathol & 6.441 & \\
J Pathol & 5.333 & 0.957 \\
J Neuropath Exp Neurol & 5.037 & 1.044 \\
Am J Surg Pathol & 4.690 & 0.518 \\
Lab Invest & 3.702 & 0.445 \\
Modern Pathol & 3.643 & 0.748 \\
Human Pathol & 3.369 & 0.459 \\
& & \\
\hline
\end{tabular}

${ }^{\mathrm{a}}$ Impact Factor for 2004: the number of citations of articles published in calendar years 2002-2003, as cited in articles published in calendar year 2004.

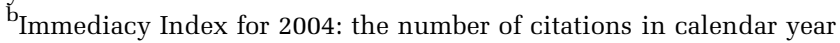
2004 of articles published in calendar year 2004.

biomedical journals. The top group can be viewed as 'high impact' journals, which publish a wide array of scientific articles for a broad audience. The second group identifies the highest impact journals in a selection of 'disease' or 'tissue/organ' categories. While this second listing is not comprehensive, one can observe that disease- or tissue/organ-specific journals carry substantial impact in their own right. It is notable, therefore, that the leading journals of the pathology literature, in the third group, fall considerably behind the leading journals of the disease- and tissue/organ-specific group.

Table 6 then gives the data for the number of articles classified as having 'pathology', as published in each of the journals listed in Table 5 during the period January 1, 2005-May 17, 2005. Although not uniform, it is clear that the higher impact journals are a welcome home for pathology-based research. Indeed, even with this limited sampling of disease- and tissue/organ-specific journals, it can be seen that investigative pathology is published substantially outside the pathology literature.

Table 7 shows the numerology of journals occupying the 10 Impact Factor intervals between 0 and 50. Fully $95 \%$ of all biomedical journals earning an 
Table 6 Pathology articles published in the medical literature ${ }^{\mathrm{a}}$

\begin{tabular}{lcc}
\hline Journal 'Pathology' articles, & Sum \\
$1 / 1 / 05-5 / 17 / 05$ &
\end{tabular}

Ann Rev Immunol

New Eng J Med

Nature

Science

Cell

J Am Med Assoc

J Exp Med

J Clin Invest

Neuron

J Natl Cancer Inst

Gastroenterology

Circulation

Hepatology

Mol Cell Proteomics

Brain

J Am Soc Nephrol

Am J Pathol

J Pathol

Am J Surg Pathol

J Neuropath Exp Neurol

Lab Invest

Human Pathol

Mod Pathol

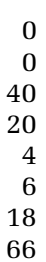

\section{0}

40

40

4

4
36
48
4
36
1
48
1

63

37

77

34

25

59

aThe term 'pathology' was crossindexed to the journal titles, for the period January 1, 2005-May 17, 2005. Journals selected are the examples given in Table 5.

Table 7 Number of journals in each Impact Factor range (2004 data)

\begin{tabular}{lr}
\hline Impact factor range & \# Journals \\
\hline $50-55$ & 1 \\
$45-50$ & 0 \\
$40-45$ & 1 \\
$35-40$ & 2 \\
$30-35$ & 8 \\
$25-30$ & 2 \\
$20-25$ & 12 \\
$15-20$ & 18 \\
$10-15$ & 49 \\
$5-10$ & 191 \\
$0-5$ & 5,635 \\
\end{tabular}

Impact Factor rank, fall at 5 or below. For the 2004 Impact Factors, only two 'Pathology' journals are above this arbitrary line.

These publication data are given without strong editorial commentary. Only two simple interpretations are offered. First, a substantial proportion of pathology research is published in journals targeting specific scientific constituencies. Second, a substantial proportion of pathology research is targeted towards the highest impact journals, with success. We hope that it does not appear too parochial to aspire to the publication of 'the best' in pathology research within the pages of pathology-oriented journals. We hold to the view that there is ample opportunity for growth and visibility of the several pathology journals, not at the expense of one another, but rather to the enhancement of the reputation of research conducted by academic pathologists.

\section{Conclusion}

Academic Pathology is a key player in human molecular science and in the powerful initiatives of the National Institutes of Health, but will remain so only if we are aggressively proactive in managing our resources. We have entered the era of massive data generation, and must restructure our thinking about data, hypothesis testing, and sharing. Pathology is ideally positioned to navigate the multidimensional space of (human tissuemolecular analysis-clinical data), so as to advance understanding of disease pathogenesis; identify molecular targets for intervention; and determine clinical outcomes of both molecular expression and targeting. We also consider that our own specialty journals could be the place to drive this agenda. It is our earnest hope that Academic Pathology can play a leading role in the remarkable biomedical advances to be made as the 21st century unfolds.

\section{Acknowledgements}

Our thanks are expressed to the following individuals, whose innovative thinking and exemplary work have helped the authors in articulating the ideas presented in this editorial: Leo T Furcht, MD, Julie A Schneider, DPhil, Jules J Berman, MD, Stephen J Qualman, MD, Carolyn Compton, MD, and Jose Quintans, MD. These individuals have each spoken at the annual summer meeting of the Association of Pathology Chairs, Dr Furcht in 2000 (Boulder, CO, USA), and the remainder (including the authors) at a symposium of this name at the 2004 meeting (Mont Tremblant, Quebec, Canada). Thanks also are given to Dr Schneider for her critical review of this manuscript.

\section{References}

1 Scripps Howard News Service. July 11, 2004.

2 Birmingham K. An inauspicious start for the US National Biospecimen Network. J Clin Invest 2004; 113:320.

3 http:/www.ndoc.org/about_ndc/reports/pdfs/FINAL_ NBN_Blueprint.pdf; reference source Julie Schneider, DPhil; Technology Program Manager, NCI.

4 Buetow KH. Cyberinfrastructure: empowering a 'third way' in biomedical research. Science 2005;308:821-824.

5 Zha H, Raffeld M, Charboneau L, et al. Similarities of prosurvival signals in Bcl-2-positive and Bcl-2-negative 
follicular lymphomas identified by reverse phase protein microarray. Lab Invest 2004;84:235-244.

6 Rhoden KJ, Johnson C, Brandao G, et al. Real-time quantitative RT-PCR identifies distinct c-RET, RET/ PTC1 and RET/PTC3 expression patterns in papillary thyroid carcinoma. Lab Invest 2004;84:1557-1570.

7 Beckner ME, Chen X, An J, et al. Proteomic characterization of harvested pseudopodia with differential gel electrophoresis and specific antibodies. Lab Invest 2005;85:316-327.
8 Tuziak T, Jeong J, Majewski T, et al. High-resolution whole-organ mapping with SNPs and its significance to early events of carcinogenesis. Lab Invest 2005;85: 689-701.

9 Tanami H, Tsuda $\mathrm{H}$, Okabe $\mathrm{S}$, et al. Involvement of Cyclin D3 in liver metastasis of colorectal cancer, revealed by genome-wide copy-number analysis. Lab Invest 2005;85, print copy in press 2005 June. Available from http://www.nature.com/labinvest/journal/vaop/ ncurrent/full/3700312a.html 